\title{
Poetry Slam: Heterogen und mehrsprachig im literarischen Feld
}

\section{Poetry Slam: Heterogeneous and Multilingual in the Literary Field}

\author{
Johann Georg Lughofer
}

\begin{abstract}
The article introduces Poetry Slam as a new trend in the literary field. The German-speaking slam scene is presented, with a focus on Austria and showing how poetry slam influences and changes the literary scene. In addition to performance, heterogeneity and multilingualism are defining characteristics in slam. This is not mere coincidence resulting from slam's loose rules. Rather, as the article shows, these traits are deeply inscribed in the slam movement and intrinsic to the system.
\end{abstract}

\section{Keywords}

Performance literature; youth literature; contemporary literature; intercultural literature; transcultural literature

Dieser Aufsatz wurde an der Philosophischen Fakultät der Universität in Ljubljana geschrieben im Rahmen des Forschungsprojekts "Mountaineering Literatur: Slovenia and Beyond" (J6-1808) und des Forschungsprogramms "Intercultural Literacy Studies" (P6-0265), die von der Slowenischen Forschungagentur finanziert sind. 


\section{Das Erfolgsformat Poetry Slam und seine Geschichte im literarischen Feld'}

Der Erfolg der Poetry Slam-Bewegung ist nicht mehr zu leugnen. Der nahezu globale Durchbruch gelang dem Format in nur zwei Jahrzehnten. Erst 1986 entstand Poetry Slam in der kulturaffinen Clubszene der USA; Marc Kelly Smith erfand das Format für den legendären „Green Mill Jazz Club“ in Chicago. Der Dichter und ehemalige Bauarbeiter erklärte anlässlich seines Besuchs beim Jubiläum des seit 1996 existierenden Münchner „Substanz Poetry Slams“:

Ich sog alles auf, was ich bekommen konnte. Und dies nicht aus Bildungsgründen. Mich interessierte vor allem der Umgang mit Sprache und die damit verbundenen Gefühle, die ein Gedicht bei mir und anderen auszulösen in der Lage war. Was ich jedoch niemals verstehen konnte, war die Art der Textvorträge und die Orte, an denen sie abgehalten wurden. Wie konnte jemand etwas, was ihm offensichtlich wichtig erschien, derart schlecht vorbereitet vortragen, und dieses dann auch noch häufig in der sterilen Atmosphäre eines nüchternen weißen Raums mit Stuhlreihen, ohne Bar, ohne flairerzeugende Beleuchtung, ohne die Möglichkeit, den Rest des Abends angenehm miteinander verbringen zu können. Hier hinkte die Dichtkunst den von mir ebenfalls geliebten Jazz- und Blueskonzerten in Chicagos Clubs noch um Meilen hinterher. Die Zeit machte neue Schritte nötig. ${ }^{2}$

Poetry Slam trat also von Anfang an ins Feld der Literatur mit dem Anspruch, die Spielregeln zu erneuern. Die Lesungen mit Wasserglas und Leselampe wurden als langweilig, steril und enthoben abgetan und lebendige Performances gefordert. Im „Nuyorican Poets Cafe“ konnte Bob Holman das Format weiter etablieren. 1991 überzeugte er den zentralen Fernsehkanal der Jugendkultur davon, mit MTV Poetry Unplugged den Slam noch bekannter zu machen: ein globaler Trend nahm damit seinen Anfang. In den 1990ern gab es bereits in vielen Staaten der USA, in Kanada, Europa, Japan und Südafrika Poetry Slams.

Die deutschsprachige Szene wurde in mancherlei Hinsicht die größte Slamszene der Welt. So gab es dort weltweit die größten singulären Slamereignisse: Die „Deutschsprachigen Poetry Slam-Meisterschaften“ generieren schon jährlich eine Zuhörerschaft in den Tausenden. Das Halbfinale der Meisterschaften 2019 in Berlin fand immerhin im Admiralspalast und das Finale im Tempodrom mit 3500 Sitzplätzen statt. Die zumeist jungen Besucher waren dafür bereit, für den Eintritt bis zu $€ 40$ hinzublättern. Auf Hamburgs Rennbahn in Bahrenfeld konnte 2015 der Rekord mit mehr als 5000 Zuse-

1 Die einführenden Absätze sind eine Weiterführung und Bearbeitung der Einleitung in Lughofer, Johann Georg (2021): Südöstlicher Wind in der deutschsprachigen Poetry Slam-Szene. In: Car, Milka [et al.] (hrsg.): Grenzgänge. Transkulturalität als Literatur- und Wissenschaftsform. Festschrift für Marijan Bobinac. Zagreber Germanistische Beiträge. Beiheft 10, im Druck.

2 Poetry Slam Erlangen (2002): Phänomen Poetry Slam. Ursprung und Geschichte. https://www.e-poetry. de/2002/02/phaenomen-poetry-slam-ursprung-und-geschichte/ (2. 12. 2020). 
hern bei einem Open Air-Slam aufgestellt werden. ${ }^{3}$ Der längste Poetry Slam wiederum fand übrigens im darauffolgenden Jahr in Salzburg statt und dauerte 28 Stunden. ${ }^{4}$ Auch der nach eigenen Angaben größte Slam Poetry-Verlag weltweit sitzt in Paderborn. (Slam Poetry sind übrigens die Texte für das Veranstaltungsformat Poetry Slam.)

Dazu kommt, dass Poetry Slam im deutschsprachigen Raum weiter an Bedeutung zunimmt. So werden in mehr und mehr Großstädten regelmäßig solche Ereignisse veranstaltet. In Deutschland hatte das Zeitmagazin schon vor Jahren über 700 Slams gezählt. ${ }^{5}$ In der Schweiz verzeichnet das Portal poetryslam.ch wöchentlich eine stattliche Zahl von solchen Abenden. ${ }^{6}$ In Österreich werden ebenso über 50 regelmäßig stattfindende Slamveranstaltungen genannt, zwölf davon alleine in Wien. ${ }^{7}$ Diese haben den intimen Cluboder Kulturcaférahmen schon lange gesprengt. Beispielsweise in Österreich finden in der Linzer Tabakfabrik, der Innsbrucker Kulturbackstube oder in Grazer Hörsälen Slams mit monatlich mehreren Hundert Besuchern statt. Auch der Berliner Admiralspalast, die Hamburger Elbphilharmonie, das Wiener Burgtheater oder die Kammerspiele München öffnen die Pforten regelmäßig für Poetry Slams. In den Vereinigten Staaten mögen die Slams einmal eine underground-Seite gehabt haben; in Europa startete das Format von Beginn an in bürgerlich-studentischer Umgebung und konnte schnell in die Gefilde honoriger Kulturgebäude Einzug halten. Natürlich gibt es daneben weiterhin Slams in gemütlichen kleineren Clubs.

Im literarischen Feld ziehen wohl keine anderen Veranstaltungen so viele Besucher*innen an - und darunter sind viele junge, was auch eine gewisse Zukunft sichert. Die Abende in den verschiedensten Spielstätten sind oftmals ausverkauft und es ist keine Ausnahme, dass viele Interessierte an den Türen abgewiesen werden müssen. Manche Kulturstätten sehen das Format als besten Weg überhaupt an, auch Jugendliche und Studierende anzusprechen. Die meistbesuchten Events mancher Literaturhäuser sind Slams; oft genügt der Platz kaum. Die Szene hat sich soweit professionalisiert und kommerzialisiert, dass mittlerweile sogar spezialisierte Agenturen zur Vermittlung und Organisation von Veranstaltungen und Workshops existieren - wie FOMP in Wien ${ }^{8}$.

Währenddessen müssen manche konventionelle Lesungsveranstalter zittern, dass die geringe Publikumszahl nicht für alle Beteiligten peinliche Ausmaße annimmt. Selbst wenn es sich um wirkliche Größen der Literaturszene handelt, kann für deren Auftritte, wenn überhaupt nur ein bescheidenes Eintrittsgeld erbeten werden. (Auch dann treten die Autor*innen zumeist mit Comiczeichner*innen oder Musiker*innen auf.)

3 Westerhaus, Sara: Weltrekord in Bahrenfeld - es war wirklich der größte Poetry Slam der Welt! https:// hamburg.mitvergnuegen.com/2015/der-groesste-poetry-slam-der-welt-ein-rekordversuch/ (4. 12. 2020).

4 Salzburgwiki.at (o.J.): Eintrag Poetry Slam Weltrekord. https://www.sn.at/wiki/Poetry_Slam_Weltrekord (2. 12. 2020).

5 Milbradt, Friederike (2016): Deutschlandkarte Poetry Slams. In: Zeitmagazin 50, 10. 12. 2015. https:// www.zeit.de/zeit-magazin/2015/50/deutschlandkarte-poetry-slams (4. 12. 2020).

6 Poetryslam.ch: Veranstaltungen. http://poetryslam.ch/events/ (4. 12. 2020).

7 Medusa, Mieze - Köhle, Markus (2013): Ping Pong Poetry. Die neuen besten Slamtexte. Wien: Milena, S. 257.

8 Fomp Vienna: Über Fomp. https://www.fomp.eu/ (4. 12. 2020). 
Dementsprechend ist das Selbstbewusstsein der Szene groß - so Markus Köhle: „Poetry Slam hat sich durchgesetzt und ist die paradigmatische Literaturveranstaltung des 21. Jahrhunderts [...] und kann aktuell durch nichts ersetzt werden." ${ }^{\text {"9 }}$

Bourdieus Entwurf eines literarischen Felds als autonome gesonderte Welt mit eigenen Gesetzen entwickelte die Slambewegung wohl nur sehr bedingt. Es handelt sich um einen neuen Teil des literarischen Felds, dem das kulturelle Kapital von weiten Teilen des traditionell literarischen Felds lange abgesprochen wurde und zum Teil heute noch wird, das aber von den Akteuren Publikum und Veranstalter*innen davon erhält. In Folge bekommen die Künstler*innen auch etwas ökonomisches Kapital.

\section{Das breite Netzwerk der Akteure im Feld}

Die institutionellen Mitspieler sind neben den Slam-Poet*innen vor allem Personen und Vereine, welche Örtlichkeiten für die Veranstaltungen zur Verfügung stellen - erstmals Kulturcafés, Kulturorte oder Clubs, nunmehr wie bereits angeführt genauso Literaturhäuser, Theater und andere zentrale Kulturräume. Mit manchen Stätten geht die persönliche Kooperation über Slam-Veranstaltungen hinaus. So werden z.B. im Literaturhaus Wien monatlich der "Super LeseClub“ von der Slam-Moderatorin Diana Köhle und einem Slammer - ehemals Didi Sommer und nunmehr David Samhaber - für junge Interessierte angeboten.

Slam als sehr wandlungs- und anpassungsfähiges Format hat in mehrerer Hinsicht einen breiten Raum im literarischen Feld eingenommen. Slam Poetry erreicht nicht nur auf der Bühne eine große Zahl von Menschen, sondern auch über das Fernsehen: Überregionale sowie lokale Sender gewähren dem Phänomen erhebliche Sendezeit. Der WDR begann schon 2007 mit wöchentlichen Übertragungen; Arte, 3sat, aber auch Sat1, ZDF und andere zogen nach.

Insbesondere dafür, dass es sich um eine präsenzabhängige Performanceliteratur handelt, erstaunt es, wie viele Slamtexte auch in eigenen Anthologien und Zeitschriften erscheinen. Ja, der Verlag Lektora in Paderborn hat sich rein dem Phänomen verschrieben. Seine Mitarbeiter gehören selbst zur Slamszene. Auch andere Verlage wie Voland\&Quist in Leipzig, Satyr in Berlin oder Ariel in Riedstadt haben ein breites Angebot an Slam Poetry in ihrem Angebot. Manche literarischen Zeitschriften bewegen sich darüber hinaus ebenso im Umfeld der Slambewegung. Noch immer bieten diese Verlage auch CDs und DVDs an, doch vor allem findet sich nun auf Videoplattformen wie youtube ein beeindruckendes Angebot an deutschsprachigen Slamaufzeichnungen, die oft Klickhäufigkeiten von Pophits erreichen. Bekannterweise wurde der Text „one day/ reckoning" von Julia Engelmann zum viralen Hit und 2014 in kürzester Zeit über fünf Millionen Mal aufgerufen. Das Phänomen Poetry Slam erreichte damit alle interessier-

9 Köhle, Markus (2019): Eine Erfolgsgeschichte mit besten Aussichten. In: Obermoser, Anna-Lena - Szanto, Henrik (hrsg.): G'scheit goschert. Die österreichische U20-Poetry-Slam-Anthologie. Paderborn: Lektora, S. 329-331, hier S. 330f. 
ten Feuilleton-Leser*innen verschiedener Generationen. Bis heute wurde der Beitrag aus dem 5. Bielefelder Hörsaal-Slam übrigens weit über 13 Millionen Mal angeklickt. ${ }^{10}$

Der Erfolg der Slambewegung im literarischen Feld kann vor allem auf die Offenheit und Niedrigschwelligkeit zurückgeführt werden. Damit unterscheidet sie sich essentiell vom etablierten Literaturbetrieb und von klassischen Veranstaltungen. Es gibt einen fließenden personalen Übergang zwischen Veranstalter*innen, Moderator*innen, professionellen Slammer*innen, regelmäßigen, seltenen und einmaligen Bühnenteilnehmenden und dem Publikum. Erfahrene Slammer*innen engagieren sich zumeist auch bei der „Szenenarbeit“ und veranstalten sowie moderieren Abende. Bei Veranstaltungen mit offenen Listen können dabei überhaupt alle Interessierten aktiv auf der Bühne teilnehmen und ihre Texte präsentieren. Regelmäßig werden an vielen Orten vor Veranstaltungen Workshops zum Einstieg für Newcomer gemacht und eigene U20-Wettbewerbe für jüngere Slammer*innen veranstaltet. Die aktive Nachwuchsarbeit wird auch noch weiter mit Stammtischen und ähnlichem gefördert.

Mit Publikumsbewertungen - einer zufälligen Jury oder Kampfapplause - sowie Kommunikation der Moderation mit den Zuseher*innen wird weitere Interaktivität und Teilhabe ermöglicht. Einzelne Texte beziehen das Publikum mit ein: lassen es z. B. Worte oder ganze Verse im Chor sprechen. Ein gelungener Slamabend hängt damit genauso vom Publikum ab wie von den Beiträger*innen auf der Bühne. Moderator*innen, altbewährte und junge Slammer*innen sowie Publikum begegnen sich also auf Augenhöhe; die Schwellen werden bewusst niedrig gehalten.

Viele Institutionen versuchen die Brückenfunktion zur Literatur für Junge fruchtbar zu machen. Jugendliche entdecken dabei ihr Interesse an Literatur und an der Arbeit mit Sprache. Dazu gibt es auch vom Slam herrührende Abendformate, welche bewusst die Hinwendung zur Literatur unterstützen sollen, etwa die Veranstaltung von Markus Köhle „Slammer. Dichter. Weiter. Konfrontieren - Reagieren - Rezitieren!“ im literarischen Quartier der Alten Schmiede in Wien. Dafür wählen ein*e Slammer*in jeweils eine*n österreichische*n Dichter*in, deren Texte sie literarisch beantworten. Die - zumeist beim jungen Publikum - weniger bekannten Größen der österreichischen Literatur werden damit lebendig und aufschlussreich präsentiert. Bildungsinstitute sind dabei ein weiterer Multiplikator der Szene. Für professionelle Slammer*innen sind Workshops an Schulen und bildungsnahen Institutionen eine wichtige Einnahmequelle. An den Schulen nehmen die Künstler*innen ebenso die Mehrfachrollen von Workshopleiter*innen, Slammer*innen und MCs (master of ceremony) - also die Moderation - ein. Selbst in der Auslandsgermanistik spielt Slam mittlerweile eine bedeutende Rolle. So führen die Tourneen der Slammer*innen weit über den deutschsprachigen Raum hinaus. Zumeist auf Einladung des Goethe-Instituts, der Österreichischen Kulturforen oder von Pro Helvetia performen sie ihre Literatur vor internationalen Zuhörer*innen und leiten genauso Workshops für Germanistikstudierende und Deutschlernende.

Das Erfolgsrezept des Dichterwettstreits, bei dem üblicherweise selbst verfasste Texte ohne Requisiten, Verkleidungen und durchgängige Musik innerhalb weniger Minuten

10 5. Bielefelder Hörsaal-Slam - Julia Engelmann - Campus TV 2013. https://www.youtube.com/ watch?v=DoxqZWvt7g8 (26. 10. 2020). 
vorgetragen werden, wird mittlerweile abgeändert in viele weitere Formate übernommen. Der Science Slam ist als Nachwuchsveranstaltung für größere Universitätsfeiern und Kongresse heute fast ein Muss. In Theatern messen sich Schauspieler*innen, die kanonisierten Texte vortragen, mit Slammer*innen in Dead or Alive-Slams. Gebärde-, Singer-Songwriter-, Accessoire-Slams und viele andere werden veranstaltet.

Insgesamt handelt es sich also nicht um ein Feld als einen relativ geschlossenen Bereich, sondern um einen sehr offenen Feldteil. Das Phänomen zeugt von der permanenten Auseinandersetzung, dem antagonistischen Charakter und der internen Dynamik des literarischen Feldes. In diesem Kampffeld behauptet sich Poetry Slam gegenüber traditionelleren Formen mit großem Erfolg - verdrängt und beeinflusst sie: Aufgrund des erfolgreichen Formats sind auch traditionelle Literaturveranstaltungen wie Lesungen zu größerer Lebhaftigkeit gezwungen. Mittlerweile konnte sich die Slamszene sicher einen breiten und unverwechselbaren Platz im Feld sichern.

\section{Kulturelles und ökonomisches Kapital}

Wenn wohl auch nur wenige Slammer*innen so zu seriösem ökonomischen Kapital kommen, gibt es doch manche. Mehrere können von Slam und dessen Verbreitung zumindest gut leben. Vor allem beweist sich Poetry Slam als Sprungbrett für junge Leute, die in die Felder der Literatur, des Kabaretts oder der Musik einsteigen wollen. Eine bedeutende Rolle im literarischen Feld ist der Dienst als Nachwuchsschule. So haben anerkannte Autor*innen wie Nora Gomringer oder Michael Lentz mit Slam ihre Karriere begonnen. Nora Gomringer war wohl als Tochter Eugen Gomringers, des Begründers der Konkreten Poesie, der Literaturbezug in die Wiege gelegt; doch bis heute pflegt sie eine vom Slam her rührende performancestarke Lyrikpräsentation und erhielt so neben vielen anderen Preisen und Stipendien 2015 den Bachmannpreis. Michael Lentz war 1998 der zweite deutsche Poetry Slam Meister überhaupt, erhielt drei Jahre später ebenso den Bachmannpreis und bekam 2006 den Ruf zum Professor an das Deutsche Literaturinstitut Leipzig, wo er mittlerweile auch die Direktion innehatte. In Österreich können junge Autor*innen wie Elias Hirschl, Ann Cotten oder Robert Prosser genannt werden, die aus dem Poetry Slam kommen. Die österreichischen Slam-Pioniere*innen Markus Köhle und Mieze Medusa behaupten sich ebenso in anderen Literaturformaten. Für die Schweiz kann man hier arrivierte und preisgekrönte Autor*innen wie Melinda Nadj Abonji, Raphael Urweider und Jürg Halter nennen.

Die bereits erwähnte Julia Engelmann nutzte ihren viralen Hit auf YouTube auch für Tourneen mit Poesie und Gitarre - und gibt ebenso regelmäßig Bestsellerbücher heraus. Als Sprungbrett für Karrieren erweist sich Slam vor allem auch für - oft literaturnahes Kabarett und andere Comedy-Formen. Bekannte Namen wären dabei die kontroverse Lisa Eckhart, Hazel Brugger, Jan Philipp Zymny, Nico Semsrott, Marc-Uwe Kling, David Scheid oder Paul Pizzera, der mit seinem Partner Otto Jaus mit rockigen Mundartsongs auch die österreichische Hitparade stürmt. Yasmin Hafedh und Mieze Medusa wiederum reüssieren mit ihrer Rapmusik, die der Spoken Word-Kunst vieler Slam Poet*innen nahesteht. 
Es lässt sich also nicht nur für einige ökonomisches Kapitel beim Slam ansammeln, vor allem ist es kulturelles Kapital, das über das Feld der Literatur hinausgetreten, oft sogar mehr in einem breiten Feld der Kunst und Unterhaltung anerkannt ist. Da dieses nicht von allen Feldteilen zu gleichen Maßen anerkannt ist, grenzen sich manche etablierteren Künstler*innen oftmals von ihrer eigenen früheren Tätigkeit bei Slams ab, die dann zum Teil als Jugendsünden abgetan werden. Augenzwinkernd spricht Hazel Brugger von den „Para-Olympics der Literatur“. ${ }^{11}$ Ein deutscher Slammer der ersten Stunde, nunmehr AfD-Politiker, Boris Preckwitz, wandte sich ebenso ab und konstatiert polemisch, dass sich Poetry Slam „mehr und mehr zu einer kulturellen Farce, in der Wettbewerbsdogma, Performancezwang und Herdentrieb einander beflügeln“ entwickelt hat. ${ }^{12}$ Seriöser kritisiert der ehemalige Slammer Sebastian Krämer die heutige Verschulung: „Einen Sport daraus zu machen, den Leute wirklich ernst nehmen - so war das nicht gemeint. Das ist überhaupt nicht der Gedanke von Bob Holman gewesen, einem der Gründer des Poetry-Slams: Wir machen hier eine Satire auf den Kulturbetrieb und wir zeigen an dieser Abstimmung, an dieser Jury-Entscheidung, dass es NICHT geht. So ein Leitsatz war ja: Der beste Text gewinnt nie. Damit war gemeint: Der Slam soll am Ende erweisen, dass Lyrik nicht für den Leistungswettbewerb ist. [...] Dann muss es natürlich auch so laufen, dass das Ganze sich immer wieder Abend für Abend ad absurdum führt und nicht, dass man tatsächlich immer ernsthaft da Stars aufbaut. [...] Das ist im Grunde alles ganz grauenvoll." ${ }^{13}$

Der Wettkampf im Feld ist dabei selbstverständlich ein ganz anderer als der gemeinte Wettkampf auf der Bühne. Die verschiedenen Textsorten und Schreibzugänge stehen nicht untereinander in Konkurrenz - wie in Bourdieus Idee der Avantgarden im Feld. Erfahrene Slammer*innen wissen, Startnummer sowie Zusammensetzung der Texte und Beiträger*innen an einem Abend sind zumeist wichtiger als die eigene Performance und Textqualität. Doch selbst wenn sie die Bedeutung des Siegens herunterspielen, lässt sich diese nicht leugnen. Finalteilnahmen und Siege - insbesondere bei überregionalen Wettbewerben - beflügeln eine Karriere, die eben über die Slamszene hinausreichen kann. Sie zieren die Kurzbiographien und bringen ganz konkret Aufmerksamkeit, weitere - eventuell gut bezahlte - Einladungen und Folgeauftritte.

\section{Heterogenität als zentrales Merkmal}

Dieser casting-ähnliche Wettbewerb führt selbstverständlich auch zur Kritik - genau wie die Absenz jeglicher Qualitätskontrolle oder die Eventisierung für eine Unterhaltungs-, Konsum- und Spaßgesellschaft. (Inwiefern Publikationen auf Papier bei allen Verlagen

11 Hugendick, David (2017): Hazel Brugger. Komik als Kampftechnik. In: Die Zeit. 14. September 2017. https://www.zeit.de/kultur/2017-09/hazel-brugger-comedy-kabarett-heuteshow-schweiz (4. 12. 2020).

12 Preckwitz, Boris (2012): Poetry Slams: Mehr und mehr eine Farce. In: Süddeutsche Zeitung, 9. 11. 2012. https://www.sueddeutsche.de/kultur/poetry-slams-mehr-und-mehr-eine-farce-1.1518545 (4. 12. 2020).

13 Zit. nach Carnal, Marc: In fünf Schritten zur Poetry Slam-Karriere. In: fm 4. 30. 4. 2017. https://fm4.orf. at/stories/2840253/ (4. 12. 2020). 
und Zeitschriften oder auf literarischen online-Portalen eine effektivere Qualitätskontrolle haben, sei dahingestellt.) Es ist wohl eine der spannungsreichen Seiten des Slams, dass sehr verschiedenartige Texte und Performances, sehr unterschiedliche Qualitäten nebeneinander auf die Bühne kommen und zumeist einem wohlwollenden Publikum begegnen. Seichte Unterhaltung und triviale Texte können direkt vor oder nach sprachlich ausgefeilten Kompositionen und hochintelligenten Reflexionen stehen.

Insgesamt steht das Format zwischen Schriftlichkeit und Mündlichkeit. Damit entspricht es nicht nur der heute äußerst wichtigen Kommunikation in sozialen Medien, sondern es ist offen in Richtung verschiedener und weiterer Felder: hin zu Fernseh- und Radiomedien, Verlagen, Veranstaltungsräumen. Wie erwähnt wurden verschiedene Sonderformate in Richtung Theater oder Universität entwickelt, es gibt genauso viele Spezialisierungen hinsichtlich der Textsorten oder Themen wie Comedy-, Rap-, Storyteller-, Haiku-, Dialekt-, Erotik- oder Politslams.

Doch im allgemeinen könnten die Textgattungen während Slamveranstaltungen unterschiedlicher kaum sein: Lyrik, Lautpoesie, Spoken Word und Rap existieren nebeneinander, sowie Aufzählungstexte, Kurzgeschichten und andere Prosa, Minidramen, kabarettartige Beiträge bis hin zum blödelnden Comedy-Text. Kritische Anklagen und politische Aufrufe sind ebenso zu hören, wie (mitunter ironische) Lobeshymnen. Diese Heterogenität ist nicht nur Zufall aufgrund der wenigen Bestimmungen für Slamtexte, sondern auch bedeutend für einen gelungenen Abend. Geübte Slammer*innen mit einem größeren Repertoire an Texten werden nach einem lustig-heiteren Text mit Sprachspielen keinen ebensolchen folgen lassen, sondern einen Kontrast wählen, was den Abend bunter und vielfältiger macht.

Ebenso die Themen umfassen die denkbar größte Reichweite: Befindlichkeitstexte und Liebesthemen finden sich neben Alltags- oder Science Fiction-Geschichten, Stellungsnahmen zu politischen oder gesellschaftlichen Fragestellungen. Dabei können die Texte von Auftritt zu Auftritt modifiziert und aktualisiert werden. Tagespolitische Neuigkeiten können genauso eingearbeitet werden wie die Stadt des Auftrittes und das aktuelle Publikum oder die bereits vorgetragenen Texte der jeweiligen Veranstaltung.

Die Heterogenität der Poetry Slams und Slam Poetry ist mit alledem beeindruckend und hat sicher einen erheblichen Anteil daran, dass man Klaus Kastbergers allgemeinem Urteil zustimmen kann: „Eine so lebendige und heterogene Szene des Schreibens wie heute hat es in der Geschichte der deutschsprachigen Literatur niemals zuvor gegeben." ${ }^{14}$

\section{Mehrsprachigkeit im Poetry Slam}

Die anfängliche deutsche Poetry Slam-Szene war durch junge, weiße, aus bürgerlichen Häusern stammende Männer, zumeist Studenten, dominiert. Das hat sich grundlegend geändert. Frauen und Performer*innen mit verschiedensten Hintergründen sind heute genauso prägend - als Veranstalter*innen sowie Slammer*innen.

14 Kastberger, Klaus (2019): Schluss mit dem Totentanz-Gerede. In: Die Zeit. 13. 2. 2017. https://www.zeit. de/kultur/literatur/2017-02/germanistik-literatur-deutsche-sprache-krise/komplettansicht (4. 12. 2020). 
Die Heterogenität betrifft vor allem eine wahrnehmbare Transkulturalität und Mehrsprachigkeit der Texte und Teilnehmenden. Dies trifft in besonderem Maße auf Slam Poetry zu, wenn auch im übrigen deutschsprachigen literarischen Feld inter- oder transkulturelle Literatur, Migrationsliteratur - oder welche Begrifflichkeit man immer wählen will - seit mindestens 2010 sehr gut repräsentiert ist. Damals wurde viel Aufmerksamkeit auf die sogenannte Migrationsliteratur gelenkt, als Melinda Nadj Abonji erstmals mit einem Buch den Deutschen sowie den Schweizer Buchpreis erhielt. Im gleichen Jahr wurde beispielsweise beim großen Kongress der Internationalen Vereinigung für Germanistik in Warschau Yoko Tawada mit genauso vielen Referaten bedacht wie die üblichen Spitzenreiter Johann Wolfgang von Goethe oder Thomas Mann. Bis heute freuen sich wohl alle Verlage, wenn sie einen möglichst exotischen Geburtsort fern von Zentraleuropa oder eine Fluchtgeschichte ihrer deutsch schreibenden Autor*innen anführen können.

Bei Slams ist diese Vielfalt nicht Mode, sondern systemimmanent. Poetry Slams wurden so ein besonderer Hort der Mehrsprachigkeit. Ganz allgemein gibt es keine Sprachvorgaben. Selbst bei den Meisterschaften ist die Bezeichnung „deutschsprachig“ nur geographisch gemeint - und alle Sprachen sind erlaubt. Immer mehr Slams sind auch bewusst mehrsprachig angelegt. CrossKultur-Slams oder i,Slam, ein muslimischer Slam, tourten so bereits durch Deutschland.

Die Niedrigschwelligkeit der Slams ist besonders dazu geeignet, junge heterogene Künstler*innen anzuziehen. Slam erwies sich so als adäquates Sprachrohr für Künstler*innen mit verschiedenen Sprach- und Migrationshintergründen. Es ist wohl für viele ein großer Unterschied, einen Beitrag für eine Buch- oder Zeitschriftenpublikation vorzubereiten oder einen kurzen Text für den Bühnenvortrag am Abend zu verfassen. Auch das studentische, tendenziell linksliberale Publikum ist wohl besonders offen hinsichtlich Fluchtgeschichten, Transkulturalität und Mehrsprachigkeit. Damit ist Slam ein bedeutendes Eintrittstor ins literarische Feld.

Exemplarisch sollen ein paar solche Stimmen vorgestellt werden. Yasmin Hafedh wehrt sich mit ihrem Text „Wo kommst du her?" 15 gegen die Einordnung in das Label Migrationsabstammung. Sie beschäftigt sich aber durchaus mit Interkulturalität und am 23. Oktober 2018 führte so die Künstlerin auch durch den 5. C3-Slam im Rahmen der Veranstaltungsreihe Bildung im Centrum für Internationale Entwicklung in Wien, welcher dem Thema Mehrsprachigkeit gewidmet war. ${ }^{16}$ Henrik Szanto, mit ungarischer und finnischer Abstammung in Deutschland aufgewachsen und mittlerweile in Wien lebend, reflektiert dabei in einem lustigen Text über finnische Redewendungen und präsentiert unterhaltsame Sprachvergleiche ausgehend vom Lateinunterricht. Einen bereits vorgetragenen Text liest er erneut - aber in der Form, nachdem dieser in der Übersetzungsmaschine ins Maori und danach zurück ins Deutsche übertragen wurde. Das amüsante Resultat ist per se eine Reflexion der Übersetzungen von Menschen und künstlicher

15 Yasmin Hafedh - Wo kommst du her. https://www.youtube.com/watch?v=9Z13fNQmiCo (4. 12. 2020).

16 Dieses Slamereignis findet sich in voller Länge auf youtube: Bühne frei für eure Stimmen! Viele Sprachen - ein Mikro. Der Poetry Slam der Mehrsprachigkeit. https://www.youtube.com/watch?v=_HgJFgHXww4 (4. 12. 2020). 
Intelligenz. Mit sehr unterschiedlichen Zugangsweisen und auch verschiedenen Textsorten folgen junge Beiträger*innen vor allem aus dem vorhergegangenen Workshop. Eine erst kurz in Österreich lebende Afghanin erzählt über Bombenanschläge in der Heimat und rezitiert nachdenkliche Gedichte auf Deutsch und Persisch; ein junger Niederländer übersetzt sein niederländisch geschriebenes Gedicht ins Deutsche und Englische und präsentiert einen Rap in seiner Erstsprache. Eine junge Österreicherin reflektiert ausgehend von einem Erasmusaufenthalt - und Fragen in Italienisch, Englisch und Deutsch - über Zukunftspläne. Eine Französin präsentiert einen Textbeitrag mit französischen, türkischen und arabischen Zitaten und Deutsch im sanften französischen Akzent über einen Flüchtling und exotistische Wahrnehmungen des Anderen sowie ein rein französisches Slam Poem.

Durchwegs fremdsprachige Texte sind bei Poetry Slams möglich. Ganz normal für Slamtexte ist es, wenn Wörter aus anderen Sprachen eingestreut werden. Das betrifft wenig überraschend insbesondere das Englische. Die selbstverständliche Verwendung und Akzeptanz von neuen Anglizismen - oftmals der Jugendsprache - geht so weit, dass beispielsweise in der Anthologie G'scheit goschert die vertretenen jungen österreichischen Slammer*innen als „Rapper“, „Web-Developer“, „cool“ vorgestellt werden; sie „performen Beats“, bewahren ihren „sense of humour“, finden ihr Leben „awesome“, „keepen es gerne real“ oder „representen“ etwas. ${ }^{17}$ Übrigens sind in dieser Sammlung „österreichischer U20 Poetry Slam“ viele Südtiroler*innen vertreten, die mitunter auch in Italien aktiv sind - und so eine weitere mehrsprachige, interkulturelle und europäische Facette in das Kunstformat bringen. Außerdem findet sich darin ein englischer Text von der in Nigeria geborenen, zweifachen österreichischen U20 Vizemeisterin von 2017 und 2018 Precious Nnebedum ${ }^{18}$, die auch sonst deutsche, englische und denglische Texte vorträgt - damit übrigens nicht in ihren „native tongues Igbo or Pidgin“19.

Ebenso finden sich sprachreflexive Texte, viele im Dialekt. Auch darauf soll in Sachen Mehrsprachigkeit hingewiesen werden, dass nicht nur in Standardsprachen performt wird - sondern mit dialektalen oder Slang-Einsprengseln bzw. ganz in diesen Formen. Die innere Mehrsprachigkeit der deutschsprachigen Länder kommt also beim Slam ebenso voll zur Geltung. Viele der jungen österreichischen Nachwuchskünstler*innen kommen dabei aus der hervorragenden Grazer Slam Szene, als deren „Architekt und Konzeptionist“20 Mario Tomic aus Bosnien bzw. Deutschland gilt, der genauso deutsche wie bosnische Texte verfasst. Mit Markus Köhle performt er auch den Text „Mehrsprachigkeit is a gift" mit spanischen und englischen Elementen sowie ganzen bosnischen Textabschnitten..$^{21}$

17 Obermoser, Anna-Lena - Szanto, Henrik (2019) (hrsg.): G'scheit goschert. Die österreichische U20-Poetry-Slam-Anthologie. Paderborn: Lektora, S. 20, 32, 37, 49,

18 Nnebedum, Precious: Creations Son. In: ebd., S. 209-213.

19 Ebd., S 362.

20 Ebd., S. 287.

21 Library Slam. Markus Köhle \& Mario Tomic: Mehrsprachigkeit is a gift. https://www.youtube.com/ watch?v=exFfoBOM45Y (9. 11. 2020). 
Weitere Beispiele seien genannt: Omar Khir Alanam erzählt von seiner wohl selbst erlebten Flucht aus Damaskus in seinem Text „Heimat“. ${ }^{22}$ Zuvor erklärt er, dass er seit drei Jahren in Österreich sei und erst deutsch lerne, und entschuldigt sich für seine Aussprache, obwohl er bestens verständlich ist. Manche arabischen Wörter - Demonstrationsschreie - unterstreichen das Lokalkolorit seiner ersten Heimat sowie das politische Umfeld. Khir Alanam belegte 2017 den dritten Platz bei den Österreichischen Poetry Slam Meisterschaften, auch Buchpublikationen folgten.

Der Beispiele gibt es unzählige mehr - in der Schweiz und Deutschland zeigt sich die gleiche offene mehrsprachige Atmosphäre in der Poetry Slam-Szene. Die von Sulaiman Masomi herausgegebene, neue Anthologie Wir sind gekommen um zu schreiben. 30 grenzenlose Texte ${ }^{23}$ gibt davon beeindruckend Zeugnis. Exemplarisch sollen ein paar, auch darin vorkommende Slammer*innen vorgestellt werden.

Der „Lautpoet“ Dalibor Marković zeigte mit einem seiner bekanntesten Kurzgedichte, dass Mehrsprachigkeit mehr als gelebte Textpraxis, nämlich Thema und Form sein kann. In „Wenn ich Deutsch rede dann bemerkt man meinen deutschen Akzent gar nicht so sehr“24 werden in einer guten Minute ganze sechs Sprachen verwendet: Deutsch, Englisch, Kroatisch, Französisch, Portugiesisch und Spanisch. Ein Verständnis fällt dem Publikum nicht schwer, auch wenn es manche darin vorkommende Sprache oder Deutsch eigentlich nicht versteht. Wie es beim Poetry Slam gerne heißt: „If you don't understand the poem - feel it!" ${ }^{25}$ Wiederholungen bzw. parallele Strukturen, die in dem die Macht der Poesie reflektierenden Text eingewoben sind, machen dies möglich. Die Lautmalerei sowie die angenehme Stimme des Poeten dazu finden zumeist Gefallen.

Die in München aufgewachsene und heute in Zürich lebende Fatima Moumouni präsentierte bei der Poetry Slam Meisterschaft 2012 mehrere kurze Texte $^{26}$, in denen sie lange vor der allgemeinen Bekanntheit der Black lives matter-Bewegung Rassismus in Deutschland auf hohem reflektiven und sprachlichen Niveau thematisiert. Sie kam damals auch ins U20-Finale und wurde deutschsprachige Vizemeisterin - mit englischen Textanteilen. ${ }^{27}$ Im selben Jahr gewann sie auch die bayrische Meisterschaft in der Kategorie U20; 2016 stand sie im Finale der deutschsprachigen Meisterschaft.

Besonders häufig werden weiters Soziolekte wie die „Kanaksprak“, die durch Feridun Zaimoglu Berühmtheit erlangt hat, verhandelt. Der in Kabul geborene und in Krefeld aufgewachsene Sulaiman Masomi, der Herausgeber des genannten Sammelbands, be-

22 Omar Khir Alanam - Heimat |SlamIYC. https://www.youtube.com/watch?v=iBfiPwGc5ZM (4. 12. 2020).

23 Sulaiman Masomi (2019) (hrsg.): Wir sind gekommen um zu schreiben. 30 grenzenlose Texte. Berlin: Satyr.

24 Auf youtube ist ein Video mit weiteren kurzen Texten aus einer Aufführung in der ehrwürdigen Hamburger Elbphilharmonie. Dalibor - Wenn Ich Deutsch Rede. https://www.youtube.com/watch?v=DtVZgW8N9bo (4. 12. 2020).

25 Z.B. Steger, Christian (2018): "If you don't understand the poem - feel it“. Tokyo - Poetry Slam - Fortbildung und Workshop mit Fabian Navarro. In: Goethe-Institut Japan. https://www.goethe.de/ins/jp/de/spr/ eng/pas/pak/21315257.html (4. 12. 2020).

26 Poetry Slam Meisterschaft 2012 "Fatima Moumoini” 09/50. https://www.youtube.com/watch?v=Ts_PYsgfM4 (4. 12. 2020).

27 Poetry Slam Meisterschaft 2012 "Fatima Moumoini” U20-Finale 24/50. https://www.youtube.com/ watch?v=ODy2M8tXfpM (4. 12. 2020). 
leuchtet mit Texten wie „Ein Kanake sieht rot“28 nicht nur Stereotype und hinterfragt soziale Hierarchien, sondern bezieht sich eben auch direkt auf den bekannten literarischen Text, nämlich Kanak Sprak. 24 Mißtöne vom Rande der Gesellschaft (1995) von Feridun Zaimoglu, das provokante und äußerst erfolgreiche Buch, das die Begrifflichkeiten „Kanak Sprak“ und „Kanak“ zu geflügelten Worten hat werden lassen: als ehemalige Schimpfworte nun ins Positive gewendet. Ein anderes Beispiel wäre Julian Heuns „UBahn-Terkan und ich“, mit dem er 2007 die U20-Poetry Slam-Meisterschaften in Berlin gewann. ${ }^{29}$ Das lyrische Ich begegnet einem türkischstämmigen Mann, dessen Soziolekt im Text umgesetzt wird.

Diese Beispiele belegen wie unterschiedlich sehr diverse mehrsprachige Beiträge zustande kommen - und wie Poetry Slam hier eine Teilnahme und ebenso eine Teilhabe der mehrsprachigen Künstler*innen ermöglicht. Damit gestalten sie auch das literarische Feld neu und bringen eine neue Betonung auf Performanz, Lebendigkeit, Heterogenität und Mehrsprachigkeit.

\section{Fazit}

Poetry Slam war wohl in den letzten Jahrzehnten die bedeutendste Erneuerung im deutschsprachigen literarischen Feld. Dabei wurde die Veranstaltungspraxis im Bereich neu aufgestellt und die Slamabende haben sich einen prominenten Platz erobert - zuerst in Kulturcafès, mittlerweile ebenso in eleganten Kulturorten - von Literaturhäusern über Theater bis hin zu Opern- und Konzertgebäuden. Mit diesem Beispiel kann der Wettkampf im Feld und die interne Dynamik besonders gut illustriert werden. Dabei handelt es sich nicht nur um eine gewisse Verdrängung, sondern auch um Prägung und Änderung zumindest der Literaturveranstaltungen: für viele als langweilig empfundene Lesungen der traditionellen Art und Weise, in denen und mitunter allzusehr der eigenen Bedeutung und des Weltschmerzes bewusst vorgetragen wird, sind heute mittlerweile nur schwer denkbar - werden oftmals vom Publikum bestraft und mittlerweile auch von vielen Veranstalter*innen gemieden. Von allem Autor*innen wird nämlich heute eine lebhafte Performance gefordert.

Die Notwendigkeit eines abwechslungsreichen Abends, die Niedrigschwelligkeit, die Bedeutung des Performativen sowie die Zwischenstellung zwischen mündlicher und schriftlicher Sprachverwendung fördern formatimmanent Heterogenität und Vielfalt. Diese wurden so - sicher nicht zufällig aufgrund der lockeren Regeln - zum wesentlichen Merkmal des Poetry Slams auf allen Ebenen: sei es bei den Textsorten und Literaturzugängen, sei es bei den Teilnehmer*innen und deren Inszenierung. Dabei spielt auch Mehrsprachigkeit eine wichtige Rolle, die in diesem Format der deutschsprachigen Literatur einen prominenten Platz eingenommen hat und entsprechend wirken kann.

28 Auf youtube hat dieses Video übrigens über 700.000 Klicks! Sulaiman Masomi - Ein Kanake sieht rot. https://www.youtube.com/watch?v=lllr32BWXGs (4. 12. 2020).

29 Julian Heun, the winner of SLAM U20 2007. https://www.youtube.com/watch?v=unkkGyVk4N4 (4. 12. 2020). 


\section{Literatur}

Allocca, Daniela (2019): One Day - werden die Meistersinger wiederkommen: Poetry Slam oder die Wirkung des Performativen. In: Di Rosa, Valentina - Röhnert, Jan (hrsg.): Im Hier und Jetzt. Konstellationen der Gegenwart in der deutschsprachigen Literatur seit 2000. Köln: Böhlau, S. 223-236.

Anders, Petra (2012): Intermedialität der Slam Poetry. In: Bathrick, David - Preußer, Heinz-Peter (hrsg.): Amsterdamer Beiträge zur neueren Germanistik. Literatur inter- und transmedial. Vol. 82(1), Amsterdam/ New York: Brill, S. 281-310.

Böttcher, Bas - Hogekamp, Wolf (2020) (hrsg.): Die Poetry-Slam-Fibel 2.0: 25 Jahre Werkstatt der Sprache. Berlin: Satyr.

Bourdieu, Pierre (1993) : Soziologische Fragen. Frankfurt am Main: Suhrkamp.

Bourdieu, Pierre - Wacquant, Loic J.D. (1996) : Reflexive Anthropologie. Frankfurt am Main: Suhrkamp.

Bourdieu, Pierre (2001): Meditationen. Zur Kritik der scholastischen Vernunft. Frankfurt am Main: Suhrkamp.

Bekes, Peter - Frederking, Volker (2009) (hrsg.): Die Poetry-Slam-Expedition: Bas Böttcher. Ein Text-, Hör- und Filmbuch. Braunschweig: Schroedel.

Carnal, Marc: In fünf Schritten zur Poetry Slam-Karriere. In: fm 4. 30. 4. 2017. https://fm4.orf. at/stories/2840253/ (4. 12. 2020).

Ditschke, Stephan (2008): „Ich sei dichter, sagen sie“. Selbstinszenierung beim Poetry Slam. In: Grimm, Gunter E. - Schärf, Christian (hrsg.): Schrifsteller-Inszenierungen. Bielefeld: Aisthesis, S. $169-184$.

Gasteiger, Carolin (2014): Hype um Julia Engelmann. Generation Mutlos meldet sich zu Wort. In: Süddeutsche Zeitung, 21. 1. 2014. https://www.sueddeutsche.de/kultur/hype-um-julia-engelmann-generation-mutlos-meldet-sich-zu-wort-1.1867901 (4. 12. 2020).

Grothe, Simon: 20 Jahre Poetry Slam in Deutschland.Wolf Hogekamp: Vater des Wortwettbewerbs. In: Der Tagesspiegel, 17. 4. 2014. https://www.tagesspiegel.de/berlin/20-jahre-poetryslam-in-deutschland-wolf-hogekamp-vater-des-wortwettbewerbs/9775126.html (4. 12. 2020).

Heinrich, Rebecca (2018): Ich bin nicht fremd. Gesellschaftskritik in Slam-Poetry und Poetry-Slam. In: Bstieler, Michaela [et al.] (hrsg.): Kunst als gesellschaftskritisches Medium. Wissenschaftliche und künstlerische Zugänge. Bielefeld: transcript (=Image 131), S. 285-290.

Jurt, Joseph (1995): Das literarische Feld. Das Konzept Pierre Bourdieus in Theorie und Praxis. Darmstadt: Wissenschaftliche Buchgesellschaft.

Jurt, Joseph (2002): Text und Kontext. Zur Theorie des literarischen Feldes. In: Foltinek, Herbert - Leitgeb, Christoph (hrsg.): Literaturwissenschaft: intermedial - interdisziplinär. Wien: Verlag der österreichischen Akademie der Wissenschaften (=Veröffentlichungen der Kommission für Literaturwissenschaft 22), S. 97-119.

Lughofer, Johann Georg (2019): Poetry Slam - vieles, aber kein kurzfristiger Boom. In: Literatur und Kritik, November 2019, S. 33-43.

Marković, Dalibor (2016): Und sie schreiben auf Deutsch? Dresden, Leipzig: Voland \& Quist.

Masomi, Sulaiman (2012): Poetry Slam. Eine orale Kultur zwischen Tradition und Moderne. Paderborn: Lektora.

Masomi, Sulaiman (2019) (hrsg.): Wir sind gekommen um zu schreiben. 30 grenzenlose Texte. Berlin: Satyr. Medusa, Mieze - Köhle, Markus (2011): Mundpropaganda : Slam Poetry erobert die Welt. Wien: Milena. 
Medusa, Mieze - Köhle, Markus (2013): Ping Pong Poetry. Die neuen besten Slamtexte. Wien: Milena.

Medusa, Mieze - Köhle, Markus (2017) (hrsg.): Slam, Oida! 15 Jahre Poetry Slam in Österreich. Paderborn: Lektora.

Meyer-Kalkus, Reinhart (2020): Geschichte der literarischen Vortragskunst. Stuttgart: J.B. Metzler.

Milbrand, Friederike (2015): Deutschlandkarte Poetry Slams, in Zeitmagazin 50, 29. 12. 2015. https://www.zeit.de/zeit-magazin/2015/50/deutschlandkarte-poetry-slams (4. 12. 2020)

Obermoser, Anna-Lena - Szanto, Henrik (2019) (hrsg): G'scheit goschert. Die österreichische U20Poetry-Slam-Anthologie. Paderborn: Lektora.

Poetry Slam Erlangen (2002): Phänomen Poetry Slam. Ursprung und Geschichte. https://www.epoetry.de/2002/02/phaenomen-poetry-slam-ursprung-und-geschichte/ (2. 12. 2020).

Porombka, Stephan (2001): Slam, Pop und Posse. Literatur in der Eventkultur. In: Harder, Matthias (hrsg.): Bestandsaufnahmen. Deutschsprachige Literatur der neunziger Jahre aus interkultureller Sicht. Würzburg: Königshausen \& Naumann, S. 27-42.

Preckwitz, Boris (2002): Slam-Poetry - Nachhut der Moderne. Eine literarische Bewegung als AntiAvantgarde. Marburg: Tectum.

Preckwitz, Boris (2005): Spoken Word \& Poetry Slam. Kleine Schriften zur Interaktionsästhetik. Essays. Wien: Passagen.

Preckwitz, Boris (2012): Poetry Slams: Mehr und mehr eine Farce. In: Süddeutsche Zeitung, 9. 11. 2012. https://www.sueddeutsche.de/kultur/poetry-slams-mehr-und-mehr-eine-farce- 1.1518545 (4. 12. 2020).

Preckwitz, Boris (2014): Kitsch in Performance. In: EXOT. Zeitschrift für komische Literatur, 18. Berlin: Satyr, S. 94-96.

Riedel, Margit (2010): Slam Poetry - interkulturell. Zur Didaktik mündlich vorgetragener deutschsprachiger Texte. In: Temeswarer Beiträge zur Germanistik, 7, S. 35-66.

Smith, Marc Kelly - Kraynak, Joe (2004): The Complete Idiot's Guide to Poetry Slam. New York: Alpha Books.

Strack, Karsten - Serrer, Michael (2017) (hrsg.): Poetry Slam - das Handbuch. Paderborn: Lektora. Westermayr, Stefanie (2010): Poetry Slam in Deutschland. Theorie und Praxis einer multimedialen Kunstform. Marburg: Tectum.

Wirag, Lino (2014): Die Geburt des Poetry Slams aus dem Geist des Theaters. In: KulturPoetik, 14, 2. Göttingen: Vandenhoeck \& Ruprecht, S. 269-281.

Zaimoglu, Feridun (1995): Kanak Sprak. 24 Mißtöne vom Rande der Gesellschaft. Hamburg: Rotbuch.

Ass. Prof. MMag. Dr. Johann Georg Lughofer, MA / johann.lughofer@ff.uni-lj.si

Univerza v Ljubljani, Filozofska fakultetat, Oddelek za germanistiko z nederlandistiko in skandinavistiko, Aškerčeva 2, 1000 Ljubljana, SLO

www.ff.uni-lj.si 